\title{
Dynamics of a collapsing and exploding Bose-Einstein condensed vortex state
}

\author{
Sadhan K. Adhikari \\ Instituto de Física Teórica, Universidade Estadual Paulista, 01.405-900 São Paulo, São Paulo, Brazil
}

(Received 30 April 2002; published 4 October 2002)

\begin{abstract}
Using the time-dependent mean-field Gross-Pitaevskii equation we study the dynamics of small repulsive Bose-Einstein condensed vortex states of ${ }^{85} \mathrm{Rb}$ atoms in a cylindrical trap with low angular momentum $\hbar L$ per atom $(L \leqslant 6)$, when the atomic interaction is suddenly turned attractive by manipulating the external magnetic field near a Feshbach resonance. Consequently, the condensate collapses and ejects atoms via explosion and a remnant condensate with a smaller number of atoms emerges that survives for a long time. Details of this collapse and explosion are compared critically with a similar experiment performed with zero angular momentum $(L=0)$ by Donley et al. [Nature (London) 412, 295 (2001)]. A suggestion for a future experiment with the vortex state is made.
\end{abstract}

DOI: 10.1103/PhysRevA.66.043601

PACS number(s): 03.75.Fi

\section{INTRODUCTION}

The recent observation $[1,2]$ of Bose-Einstein condensates (BECs) of dilute trapped bosonic atoms with repulsive interaction at ultralow temperature has intensified theoretical and experimental studies on various aspects of the condensate [3]. One fascinating feature is the observation of quantized vortices [4] and a vortex lattice $[5,6]$ in the condensate as this is intrinsically related to the existence of superfluidity. Another interesting feature is the formation of a stable condensate composed of a finite number of attractive atoms less than a critical number $N_{\text {cr }}$ [2]. The third noteworthy feature is the observation of Feshbach resonances in ${ }^{23} \mathrm{Na}$ [7], ${ }^{85} \mathrm{Rb}$ [8], and Cs [9] atoms, as in the presence of such resonances the effective atomic interaction can be varied in a controlled fashion by an external (background) magnetic field [10].

For superfluid ${ }^{4} \mathrm{He}$ II in a rotating container, no motion of the fluid is observed below a critical rotational frequency. Above this frequency quantized vortices appear in ${ }^{4} \mathrm{He}$ II, manifesting its superfluidity. However, because of the strong interaction, the theoretical description of this system is not easy. Quantized vortices have been observed [4-6] in trapped BEC's and can be generated in theoretical mean-field models [11-17] based on the Gross-Pitaevskii (GP) [18] equation. Different ways for generating vortices in a BEC have been suggested [13], e.g., via spontaneous formation in evaporative cooling [14], via controlled excitation to an excited state [15], by stirring a BEC using a laser with an angular frequency above a critical value [12], or by the rotation of an axially symmetric trap with an angular frequency above a similar critical value [16]. In contrast to liquid ${ }^{4} \mathrm{He}$ II, a trapped BEC of small size is dilute and weakly interacting, which makes a mean-field analysis appropriate.

The observation of a condensate of attractive ${ }^{7} \mathrm{Li}$ atoms and the subsequent measurement of the critical number $N_{\mathrm{cr}}$ [2] are in good agreement with the mean-field analyses in a spherically symmetric trap [19], although the agreement is not as good in the case of ${ }^{85} \mathrm{Rb}$ [20] atoms in an axially symmetric trap [21]. If the number of atoms can somehow be increased beyond this critical number, due to interatomic attraction the condensate collapses, emitting atoms until the number of atoms is reduced below $N_{\text {cr }}$. With a supply of atoms from an external source the condensate grows again beyond $N_{\mathrm{cr}}$ and a sequence of collapses has been observed in ${ }^{7} \mathrm{Li}$ by Gerton et al. [2], where the number of atoms remains close to $N_{\text {cr }}$ and the collapse is driven by a stochastic process.

Recently, a more challenging experiment was performed by Donley et al. [22] on a BEC of ${ }^{85} \mathrm{Rb}$ atoms [8] in an axially symmetric trap, where they varied the interatomic interaction by an external magnetic field near a Feshbach resonance [10]. Consequently, they were able to change the sign of the atomic scattering length, thus transforming a repulsive condensate of ${ }^{85} \mathrm{Rb}$ atoms into a collapsing and highly explosive attractive condensate, and they studied the dynamics of the same [22]. Immediately after the jump in the scattering length, one has a highly unstable BEC, where the number of atoms could be much larger than $N_{\text {cr }}$. Donley et al. [22] provided a quantitative estimate of the explosion by measuring the number of atoms remaining in the BEC as a function of time until an equilibrium is reached. This phenomenon of emission of a very large number of atoms in a small interval of time is reminiscent of an explosion and looks very much like a tiny supernova, or exploding star.

The essential aspects of the above experiments by Gerton et al. [2] and by Donley et al. [22] have been theoretically described by a variety of authors using the GP equation [18]. The theoretical analyses not only produced time-independent results, such as the critical number $N_{\mathrm{cr}}[19,21]$, but also timedependent results, such as the variation of number of atoms of the BEC during collapse and explosion [23-28], both in reasonable agreement with experiment. This consolidates the use of the mean-field GP equation in describing the dynamics of collapsing and exploding BEC's of small to medium size. These BEC's composed of several thousand atoms can be considered dilute and weakly interacting and hence amenable to mean-field treatment. Motivated by the above success, using the GP equation we propose the numerical simulation of the dynamics of a rotating collapsing and exploding BEC with a single vortex composed of a small number (several thousands) of ${ }^{85} \mathrm{Rb}$ atoms as in the experiment [22]. We consider a single vortex state $[4,11,21]$, as appropriate for small condensates, as opposed to a vortex lattice for large condensates $[5,6,17]$. A comparison of the present results with future experiments will provide a more stringent test for the mean-field GP equation. 
In this paper we perform a mean-field analysis based on the time-dependent GP equation to understand the collapse and explosion of the attractive vortex state of ${ }^{85} \mathrm{Rb}$ atoms in an axially symmetric trap. To account for the loss of atoms from the strongly attractive condensate we include an absorptive nonlinear three-body recombination term in the GP equation. The three-body recombination rate we use in numerical simulation is the same as used in a similar study with BEC's with zero angular momentum [27] and is in agreement with previous experimental measurement [29] and theoretical calculation [30]. In the present investigation we consider the complete numerical solution of the mean-field GP equation for an axially symmetric trap as in the experiment of Donley et al. [22]. As in the experiment with nonrotating condensates, we find that, in the case of rotating vortex states, also a large number of atoms could be emitted in a small interval of time and one could have an explosion.

Throughout the present numerical simulation we make the assumption that the axial symmetry of the system is maintained. For small values of nonlinearity a dynamical quadrupole instability may cause an attractive BEC vortex state to split into two pieces that rotate around the axial direction [31]. These pieces may unite to recover the original vortex and this split-merge cycle repeats. A similar instability is known to exist for BEC's in a toroidal trap [32]. Clearly, a full three-dimensional calculation of the collapsing phenomena taking into consideration the effect of the splitting of vortex states seems to be practically impossible at present and will be a welcome future work. In view of this, here we present an axisymmetric model of the same, which is expected to provide the essentials of the collapse dynamics of the vortex states.

In Sec. II we present the theoretical model and the numerical method for its solution. In Sec. III we present our results. Finally, in Sec. IV we present a brief discussion and concluding remarks.

\section{THE GROSS-PITAEVSKII EQUATION}

\section{A. Theoretical model}

The time-dependent Bose-Einstein condensate wave function $\Psi(\mathbf{r} ; \tau)$ at position $\mathbf{r}$ and time $\tau$ allowing for atomic loss may be described by the following mean-field nonlinear GP equation $[3,18]$ :

$$
\begin{aligned}
& {\left[-i \hbar \frac{\partial}{\partial \tau}-\frac{\hbar^{2} \nabla^{2}}{2 m}+V(\mathbf{r})+\mathcal{G} N|\Psi(\mathbf{r} ; \tau)|^{2}\right.} \\
& \left.\quad-\frac{i \hbar}{2}\left[K_{2} N|\Psi(\mathbf{r} ; \tau)|^{2}+K_{3} N^{2}|\Psi(\mathbf{r} ; \tau)|^{4}\right]\right] \Psi(\mathbf{r} ; \tau)=0 .
\end{aligned}
$$

Here $m$ is the mass and $N$ the number of atoms in the condensate and $\mathcal{G}=4 \pi \hbar^{2} \mathrm{a} / \mathrm{m}$ is the strength of interatomic interaction, with $a$ the atomic scattering length. The terms $K_{2}$ and $K_{3}$ denote two-body dipolar and three-body recombination loss-rate coefficients, respectively. In Refs. [27,28] and in this paper, we have not taken into consideration. The Bose factor of $1 / 6$ in the $K_{3}$ term of Eq. (2.1) as in Santos et al.
[25] and Saito et al. [26]. There are many ways to account for the loss mechanism [23,24]. Here we simulate the atom loss via the most important quintic three-body term $K_{3}$ $[23,25,26]$. The contribution of the cubic two-body loss term [29] is expected to be negligible $[23,26]$ compared to the three-body term in the present problem of a collapsed condensate with large density, and will not be considered here.

The trap potential with cylindrical symmetry may be written as $V(\mathbf{r})=\frac{1}{2} m \omega^{2}\left(r^{2}+\lambda^{2} z^{2}\right)$ where $\omega$ is the angular frequency in the radial direction $r$ and $\lambda \omega$ that in the axial direction $z$, with $\lambda$ the aspect ratio. We are using the cylindrical coordinate system $\mathbf{r} \equiv(r, \theta, z)$ with $\theta$ the azimuthal angle. The normalization condition of the wave function is $\int d \mathbf{r}|\Psi(\mathbf{r} ; \tau)|^{2}=1$.

The GP equation can easily accommodate quantized vortex states with rotational motion of the BEC around the $z$ axis. In such a vortex the atoms flow with tangential velocity $L \hbar /(m r)$ such that each atom has quantized angular momentum $L \hbar$ along the $z$ axis. This corresponds to an angular dependence of

$$
\Psi(\mathbf{r}, \tau)=\psi(r, z, \tau) \exp (i L \theta)
$$

of the wave function, where $\exp (i L \theta)$ are the circular harmonics in two dimensions.

Now transforming to dimensionless variables defined by $x=\sqrt{2} r / l, \quad y=\sqrt{2} z / l, \quad t=\tau \omega, \quad l \equiv \sqrt{\hbar /(m \omega)}$, and

$$
\frac{\varphi(x, y ; t)}{x} \equiv \sqrt{\frac{l^{3}}{\sqrt{8}}} \psi(r, z ; \tau),
$$

we get from Eqs. (2.1) and (2.2)

$$
\begin{aligned}
& {\left[-i \frac{\partial}{\partial t}-\frac{\partial^{2}}{\partial x^{2}}+\frac{1}{x} \frac{\partial}{\partial x}-\frac{\partial^{2}}{\partial y^{2}}+\frac{L^{2}-1}{x^{2}}+\frac{1}{4}\left(x^{2}+\lambda^{2} y^{2}\right)\right.} \\
& \left.+\kappa n\left|\frac{\varphi(x, y ; t)}{x}\right|^{2}-i \xi n^{2}\left|\frac{\varphi(x, y ; t)}{x}\right|^{4}\right] \varphi(x, y ; t)=0,
\end{aligned}
$$

where $n=N a / l, \quad \kappa=8 \sqrt{2} \pi$, and $\xi=4 K_{3} /\left(a^{2} l^{4} \omega\right)$. From theoretical [33] and experimental [29] studies it has been found that, for negative $a, K_{3}$ increases rapidly as $|a|^{n}$, where the theoretical study favors $n=2$, and we represent this variation via this quadratic dependence. This makes the parameter $\xi$ above a constant [27] for an experimental setup with fixed $l$ and $\omega$, and in the present study we employ a constant $\xi$.

The normalization condition of the wave function for $K_{3}$ $=0$ is

$$
\mathcal{N}_{\text {norm }} \equiv 2 \pi \int_{0}^{\infty} \frac{d x}{x} \int_{-\infty}^{\infty} d y|\varphi(x, y ; t)|^{2}=1
$$

However, in the presence of loss $K_{3}>0, \mathcal{N}_{\text {norm }}<1$. The number of remaining atoms $N$ in the condensate is given by $N=N_{0} \mathcal{N}_{\text {norm }}$, where $N_{0}$ is the initial number. by

The root mean square (rms) sizes $x_{\mathrm{rms}}$ and $y_{\mathrm{rms}}$ are defined 


$$
\begin{aligned}
& x_{\mathrm{rms}}^{2}=\frac{2 \pi}{\mathcal{N}_{\text {norm }}} \int_{0}^{\infty} d x \int_{-\infty}^{\infty} d y|\varphi(x, y ; t)|^{2} x, \\
& y_{\mathrm{rms}}^{2}=\frac{2 \pi}{\mathcal{N}_{\text {norm }}} \int_{0}^{\infty} \frac{d x}{x} \int_{-\infty}^{\infty} d y|\varphi(x, y ; t)|^{2} y^{2} .
\end{aligned}
$$

\section{B. Calculational detail}

We solve the GP equation (2.4) numerically using a splitstep time-iteration method using the Crank-Nicholson discretization scheme [21,27,34-36]. We discretize the GP equation with time step 0.001 and space step 0.1 spanning $x$ from 0 to 15 and $y$ from -35 to 35 .

It is now appropriate to calculate the parameters of the present dimensionless GP equation (2.4) corresponding to the experiment of Donley et al. for $L=0$ [22]. As in that experiment we take the radial and axial trap frequencies to be $\nu_{\text {radial }}=17.5 \mathrm{~Hz}$ and $\nu_{\text {axial }}=6.8 \mathrm{~Hz}$, respectively, leading to $\lambda=0.389$. The harmonic oscillator length $l$ of ${ }^{85} \mathrm{Rb}$ atoms for $\omega=2 \pi \times 17.5 \mathrm{~Hz}$ and $m \approx 79176 \mathrm{MeV}$ is $l=\sqrt{\hbar /(m \omega)}$ $=26070 \AA$. One unit of time $t$ of Eq. (2.4) is $1 / \omega$ or $0.009095 \mathrm{~s}$.

We consider a stable ${ }^{85} \mathrm{Rb}$ condensate of $N_{0}=16000$ atoms with scattering length $a_{\text {initial }}=7 a_{0}, a_{0}=0.5292 \AA$. This wave function is obtained by time iteration of Eq. (2.4) employing the following normalized initial solution with a single central vortex [21]:

$$
\varphi(x, y)=\left[\frac{\lambda}{2^{2 L+3} \pi^{3}(|L| !)^{2}}\right]^{1 / 4} x^{1+|L|} e^{-\left(x^{2}+\lambda y^{2}\right) / 4}
$$

for $n=0$. In the course of the above time iteration the nonlinear parameter $n$ was increased by steps of 0.0001 until its final value was attained. Then during an interval of time 0.1 ms the scattering length was ramped to $a=a_{\text {collapse }}$. The absorptive term $\xi$ was set equal to zero throughout the above time iteration.

The final condensate is strongly attractive and unstable and undergoes a sequence of collapse and explosion. In our numerical simulation with $L \neq 0$ we consider a set of different values of $a_{\text {collapse }}\left(=-263 a_{0},-100 a_{0},-30 a_{0},-20 a_{0}\right.$, etc.) as well as $N_{0}=6000$.

For the simulation of collapse and explosion a nonzero value of $\xi(=2)$ is chosen for different $a_{\text {initial }}, a_{\text {collapse }}$, and $N_{0}$ as in Ref. [27] and the time evolution of the GP equation is continued. This value of $\xi$ reproduced the essentials of the experiment of Donley et al. [22] reasonably well for $L=0$ as well as producing [27] a $K_{3}$ in reasonable agreement with a previous experiment $\left(K_{3}=4.24 \times 10^{-25} \mathrm{~cm}^{6} / \mathrm{s}\right)$ [29] and theoretical calculation $\left(K_{3}=6.7 \times 10^{-25} \mathrm{~cm}^{6} / \mathrm{s}\right)$ [30] for $a=-370 a_{0}$. In particular we use $K_{3}=9 \times 10^{-25} \mathrm{~cm}^{6} / \mathrm{s}$ for $a=-370 a_{0}$. For smaller values of $|a|$, the $K_{3}$ values are scaled down using the relation $K_{3} \propto a^{2}$.

\section{NUMERICAL RESULT}

The numerical simulation using Eq. (2.4) with a nonzero $\xi$ as described above immediately yields the remaining num-
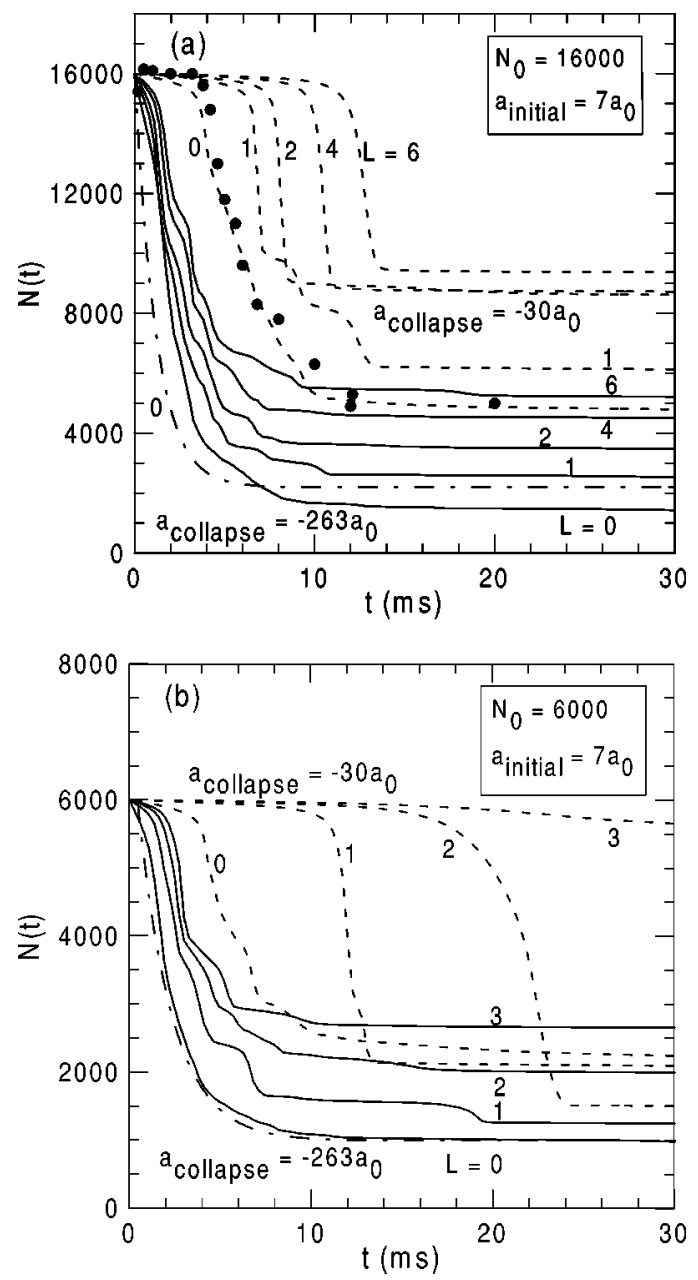

FIG. 1. The number of remaining atoms $N(t)$ in the condensate of $N_{0}=$ (a) 16000 and (b) 6000 atoms after ramping the scattering length from $a_{\text {initial }}=7 a_{0}$ to $a_{\text {collapse }}=-30 a_{0}$ (dashed line) and $-263 a_{0}$ (full line) for different $L$ as a function of time $t$. The curves are labeled by their respective $L$ values. Solid circles represent results of experiment of Donley et al. [22] for $L=0, N_{0}=16000$, and $a_{\text {collapse }}=-30 a_{0}$ and dash-dotted lines represent the average [27] over experimental results [22] for $L=0$ and $a_{\text {collapse }}=$ $-263 a_{0}$.

ber of atoms in the condensate after the jump in scattering length. The remaining number of atoms vs time is plotted in Fig. 1(a) for $a_{\text {initial }}=7 a_{0}, a_{\text {collapse }}=-30 a_{0}$, and $-263 a_{0}$, $N_{0}=16000$, and $L=0,1,2,4$, and 6. In Fig. 1(b) the same results for $N_{0}=6000$ are plotted. In this figure we also plot some results of the experiment of Donley et al. for $L=0$ $[22,27]$. These experimental results are in agreement with the simulation for $L=0$. In Fig. 2(a) we plot the particle loss curves for $N_{0}=6000, a_{\text {initial }}=7 a_{0}$ and different $a_{\text {collapse }}$ for $L=0$. In Fig. 2(b) we plot the same for $L=1$.

From Figs. 1 and 2 we find that for a fixed $N_{0}$, for a sufficiently small $L$ or a sufficiently large $\left|a_{\text {collapse }}\right|$, there could be collapse and explosion during a relatively short interval of time (called the decay time) with the loss of a large fraction of the atoms. However, there is no collapse for a large enough $L$ or a small enough $\left|a_{\text {collapse }}\right|$. For example, for $N_{0}=6000$ in Fig. 1(b) there is no collapse for $L>2$ for 

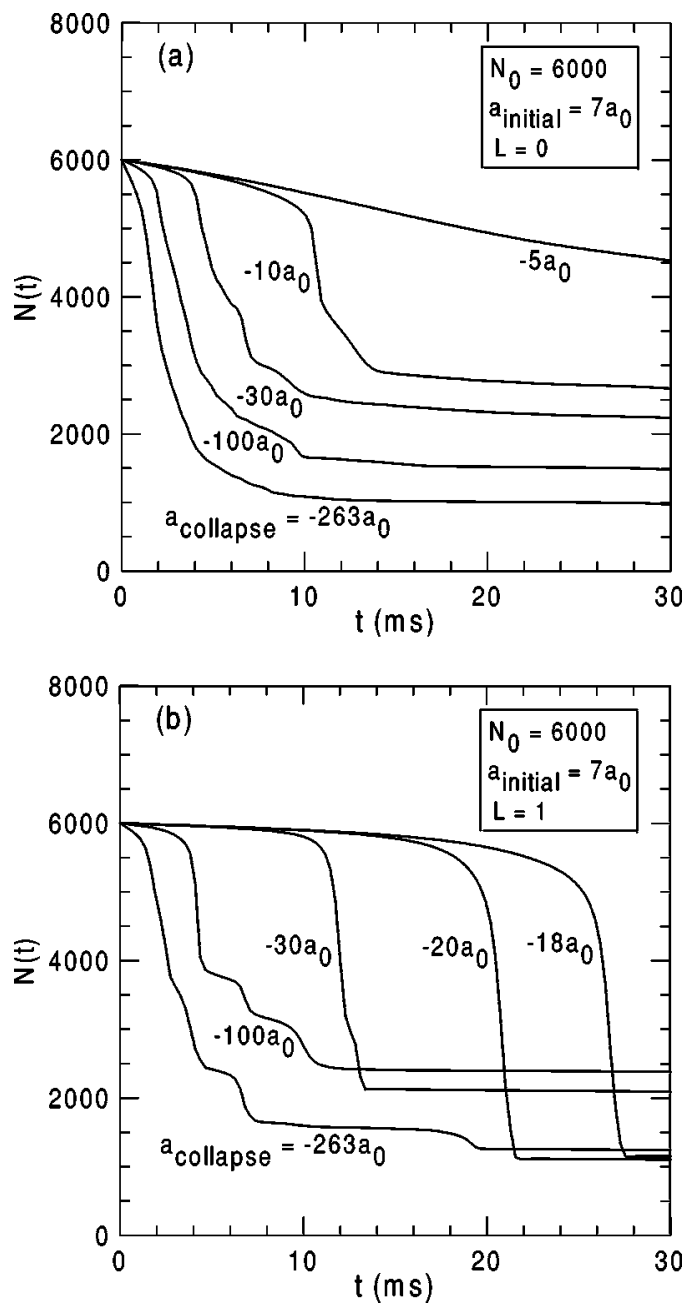

FIG. 2. The number of remaining atoms $N(t)$ in the condensate of $N_{0}=6000$ atoms for (a) $L=0$ and (b) $L=1$ after ramping the scattering length from $a_{\text {initial }}=7 a_{0}$ to different final $a_{\text {collapse }}$ as a function of time $t$. The curves are labeled by their respective $a_{\text {collapse }}$ values.

$\left|a_{\text {collapse }}\right|=30 a_{0}$ and in Fig. 2(a) there is no collapse for $\left|a_{\text {collapse }}\right|<5 a_{0}$ for $L=0$.

In the experiment of Donley et al. [22] for $L=0$ it was observed that the strongly attractive condensate after preparation remains stable with a constant number of atoms for an interval of time $t_{\text {collapse }}$, called the collapse time. This behavior is physically expected for medium to small values of $\left|a_{\text {collapse }}\right|\left(<50 a_{0}\right)$. Immediately after the jump in the scattering length to a negative value, the attractive condensate shrinks in size during $t_{\text {collapse, }}$ until the central density increases to a maximum. Then the absorptive three-body term takes full control to initiate the explosion which lasts for a few milliseconds. Consequently, the number of atoms remains constant for time $t<t_{\text {collapse }}$. The present results in Figs. 1(a) and 1(b) also show this behavior for $\left|a_{\text {collapse }}\right|$ $=30 a_{0}$. However, for larger $\left|a_{\text {collapse }}\right|\left(=263 a_{0}\right)$, the atomic attraction is very strong and the central density increases to a maximum quickly to start the explosion, and $t_{\text {collapse }}$ is close to zero. In Figs. 2(a) and 2(b) we see the dependence of particle loss and $t_{\text {collapse }}$ on $\left|a_{\text {collapse }}\right|$ for $N_{0}=6000, a_{\text {initial }}$

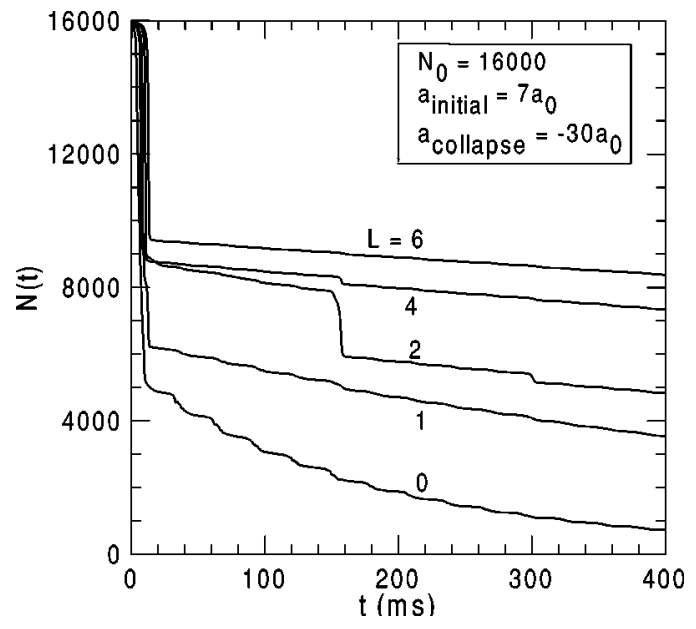

FIG. 3. The number of remaining atoms $N(t)$ in the condensate of $N_{0}=16000$ atoms for different $L$ after ramping the scattering length from $a_{\text {initial }}=7 a_{0}$ to the final $a_{\text {collapse }}=-30 a_{0}$ as a function of time $t$. The curves are labeled by their respective $L$ values.

$=7 a_{0}$, and $L=0$ and $L=1$, respectively. From Figs. 1 and 2 we find that $t_{\text {collapse }}$ increases with $L$ for a fixed $a_{\text {collapse }}$ and with $a_{\text {collapse }}$ for a fixed $L$.

From Figs. 1 and 2 we find that after the collapse the number of particles drops sharply during a small interval of time called the decay time (a few milliseconds), which means that the condensate emits a large number of particles in an explosive fashion. This emission of particles is termed the explosion.

After a sequence of collapse and explosion, for $L=0$ Donley et al. [22] observed a "remnant" condensate of $N_{\text {remnant }}$ atoms at large times containing a fraction of the initial $N_{0}$ atoms. Figures 1 and 2 show such a behavior for different values of $L$ and $a_{\text {collapse. In all cases the decay time }}$ during which the explosion takes place is small and of the order of a few milliseconds. The decay time for vortex states $(L \neq 0)$ is smaller than for nonrotating condensates $(L=0)$.

We studied the time evolution of the condensate for larger times. In Fig. 3 we plot the loss curves for $N_{0}=16000$, $a_{\text {initial }}=7 a_{0}, a_{\text {collapse }}=-30 a_{0}$, and $L=0,1,2,4,6$ at larger times. The BEC continues to lose atoms if left for a long time but at a rate much slower than during the first explosion, which we call primary. However, in their experiment Donley et al. observed that a remnant condensate containing a fraction of the atoms survived with nearly constant number for more than $1 \mathrm{~s}$. One possible reason for this discrepancy could be the following. In the actual experiment a major portion of the emitted atoms, called the burst atoms, remain trapped and oscillate around the central remnant. The presence of the burst atoms makes the measurement of the number of atoms in the remnant a difficult task [37]. Some of these burst atoms may also rejoin the remnant to compensate for the three-body loss at large times. Such an effect is not included in the present model, which, hence, presents a larger loss for the remnant compared to experiment.

We also observe an interesting phenomenon in Fig. 3, i.e., the occurrence of smaller secondary and tertiary explosions after the primary one observed for small times. For $L=2$ 


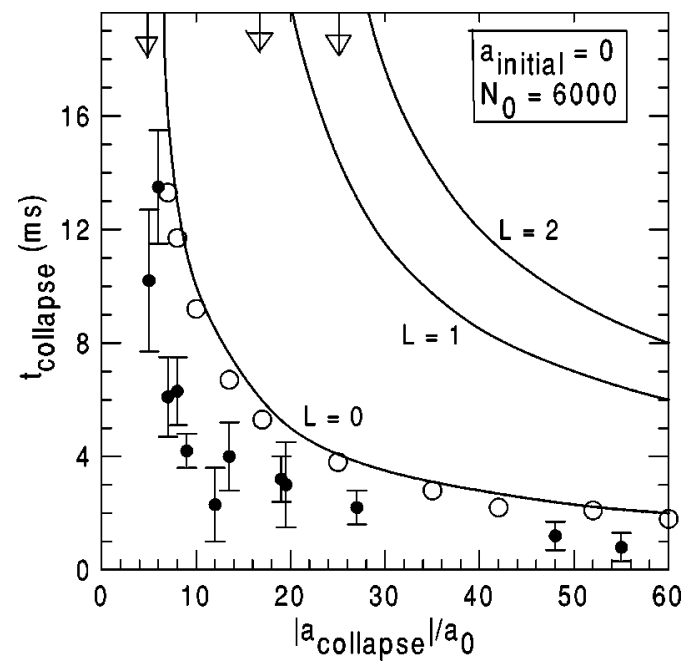

FIG. 4. The collapse time $t_{\text {collapse }}$ vs $\left|a_{\text {collapse }}\right| / a_{0}$ for $a_{\text {initial }}=0$ and $N_{0}=6000$ for different $L$. Solid circles with error bars, experiment [22] for $L=0$; open circles, axially symmetric mean-field model of Ref. [26] for $L=0$; arrows, $a_{\mathrm{cr}} / a_{0}$ values; full line, present theory for different $L$.

after the primary collapse and explosion at $t<10 \mathrm{~ms}$ with the loss of about 7000 atoms, there is another collapse and explosion with loss of about 2000 atoms at $t \approx 150 \mathrm{~ms}$. The primary and secondary explosions are separated by a large interval of time. We also see much weaker explosion(s) in the course of time evolution in Fig. 3, where the particle number varies in small steps. These explosions could be termed tertiary with the loss of a few hundred atoms. It might be interesting to see if such secondary and tertiary explosion(s) could be observed experimentally.

Donley et al. [22] provided a quantitative measurement of the variation of collapse time $t_{\text {collapse }}$ with the final scattering length $a_{\text {collapse }}$ for a given $a_{\text {initial }}=0, N_{0}=6000$, and $L=0$. We calculated this variation using our model for $L=0,1$, and 2. The $t_{\text {collapse }}$ vs $\left|a_{\text {collapse }}\right| / a_{0}$ plots for $L=0,1,2$ are exhibited in Fig. 4 and compared with experimental data for $L=0$ [22] as well as with another calculation using the mean-field GP equation in an axially symmetric trap for $L$ $=0$ [26]. We see that $t_{\text {collapse }}$ decreases with increasing $\left|a_{\text {collapse }}\right| / a_{0}$ starting from an infinite value at $\left|a_{\text {collapse }}\right|$ $=a_{\mathrm{cr}}$, where $a_{\mathrm{cr}}$ is the minimum value of $\left|a_{\text {collapse }}\right|$ that leads to collapse and explosion. The critical value $a_{\text {cr }}$ increases with $L$ and so does $t_{\text {collapse }}$ for a fixed $\left|a_{\text {collapse }}\right|$. For a given $N_{0}$, a critical value of $n \equiv n_{\text {cr }}$ can be defined via $n_{\text {cr }}$ $\equiv N_{0} a_{\text {cr }} / l$. A necessary condition for collapse is $N_{0}\left|a_{\text {collapse }}\right| / l>n_{\mathrm{cr}}[21]$. The value of $n_{\mathrm{cr}}$ for a specific case is calculated as in Ref. [21]. Consequently, $a_{\mathrm{cr}} / a_{0}$ can be obtained. The value of $a_{\mathrm{cr}} / a_{0}$ so evaluated for a specific $L$ is shown by an arrow near the curve for that particular $L$ in Fig. 4 . The $t_{\text {collapse }}$ vs $\left|a_{\text {collapse }}\right| / a_{0}$ curves should tend to infinity at the respective arrows and they do so in Fig. 4. There should not be any collapse for $\left|a_{\text {collapse }}\right|<a_{\text {cr }}$.

Donley et al. [22] measured the number of remnant atoms for $L=0, a_{\text {initial }}=7 a_{0}$ and different $N_{0}$ and $a_{\text {collapse }}$ [27]. We plot the same in Fig. 5 for $L=0,1$, and 2. The remnant number plotted in this figure is the number after the primary

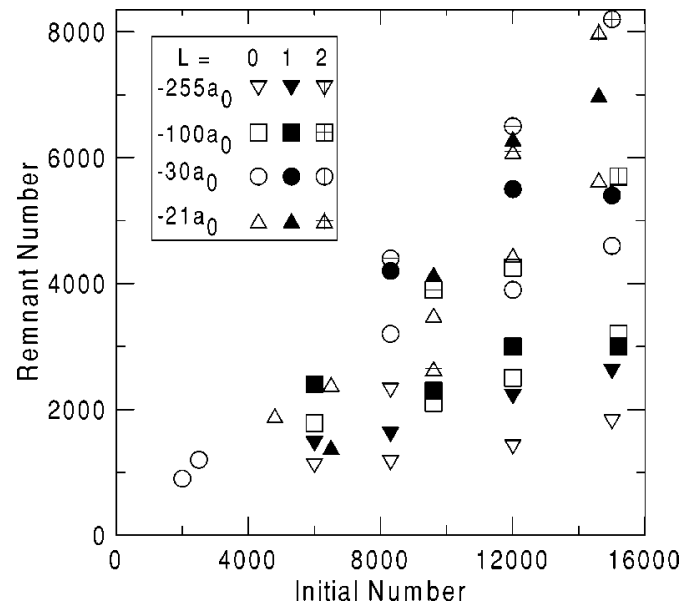

FIG. 5. Remnant number vs initial number for $a_{\text {initial }}=7 a_{0}, L$ $=0,1$, and 2, and different $a_{\text {collapse }}$. The results are represented by different types of triangle, circle, square, and inverted triangle for $L=0,1$, and 2 and $a_{\text {collapse }}=-21 a_{0},-30 a_{0},-100 a_{0}$, and $-255 a_{0}$ as indicated in the figure.

explosion(s) and not during or after possible secondary and tertiary explosions at larger times. In Figs. 1 and 2 the remnant number is obtained around $t \sim 20-30 \mathrm{~ms}$ and not at a few hundred milliseconds. Our results in Fig. 5 for $L=0$ agree well [27] with the measurements of Donley et al. [22]. In general the remnant number decreases with increasing $L$. However, there are some cases where the opposite trend has been observed in Figs. 1 and 2 as well as in Fig. 5. For the smallest values of $N_{0}$ in Fig. 5, the condensate remains stable for $L>0$, and there is no collapse and explosion and hence no remnant numbers for $L=1$ and 2. For certain $N_{0}$ the results for all three $L$ 's are not plotted as they coincide with other remnant numbers. The remnant number in some cases could be much larger than $N_{\mathrm{cr}}$ for times on the order of tens of milliseconds.

Donley et al. [22] observed that for $L=0$ the remnant condensate always oscillated in a highly excited collective state with approximate frequencies $2 \nu_{\text {axial }}$ and $2 \nu_{\text {radial }}$ being predominantly excited. This behavior emerges from the present simulation for all values of $L$. To illustrate this we plot in Fig. 6 sizes $x_{\text {rms }}$ and $y_{\text {rms }}$ vs time for the condensate after the jump in the scattering length to $-30 a_{0}$ from $7 a_{0}$ for $N_{0}=16000$ and $L=2$. We find a periodic oscillation in $x_{\mathrm{rms}}$ and $y_{\text {rms }}$ with frequencies $13.6 \mathrm{~Hz}\left(\simeq 2 \nu_{\text {axial }}\right)$ and $35 \mathrm{~Hz}$ $\left(\simeq 2 \nu_{\text {radial }}\right)$, respectively, as observed in experiment.

\section{CONCLUSION}

In conclusion, we have employed a numerical simulation based on the solution [21] of the mean-field Gross-Pitaevskii equation [18] with cylindrical symmetry to study the dynamics of collapse and explosion [22,27] of small attractive vortex states with $L>0$. The explosion is initiated by a sudden jump in the scattering length from a positive to negative value exploiting a Feshbach resonance [7-10]. In the GP equation we include a quintic three-body nonlinear recombination loss term [23-26] that accounts for the decay of the 


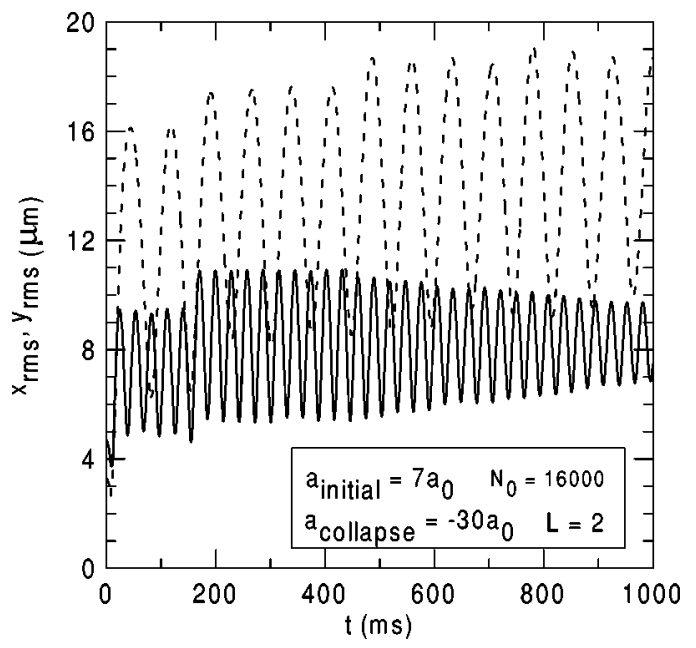

FIG. 6. The rms sizes $x_{\text {rms }}$ (full line) and $y_{\text {rms }}$ (dashed line) after the jump in the scattering length of a BEC of $16000{ }^{85} \mathrm{Rb}$ atoms for $L=2$ from $a_{\text {initial }}=7 a_{0}$ to $a_{\text {collapse }}=-30 a_{0}$ as functions of time $t$.

strongly attractive condensate. The results of the present simulation are to be considered as an extension of the experiment of Donley et al. for $L=0$ [22] to small vortex states.

We find the following features of this dynamics from the present numerical simulation. (1) The condensate undergoes collapse and explosion during a small interval of time of a few milliseconds and finally stabilizes to a remnant condensate containing a fraction of the initial number of atoms. The number in the remnant condensate for times on the order of tens of milliseconds can be much larger than the critical number for collapse $N_{\text {cr }}$ for the same atomic interaction. (2) In some cases after the primary explosion small secondary and tertiary explosions are observed. (3) The explosion takes place during a decay time of a few milliseconds. This decay time for a rotating and exploding BEC with a vortex $(L$ $>0)$ is smaller than the same for a nonrotating condensate with $L=0$. (4) The remnant condensate executes radial and axial oscillations in a highly excited collective state for a long time with frequencies $2 \nu_{\text {radial }}$ and $2 \nu_{\text {axial }}$. (5) After the sudden change in the scattering length to a large negative value, the condensate needs an interval of time $t_{\text {collapse }}$ before it experiences loss via explosion. The interval $t_{\text {collapse }}$ increases with $L$ and $a_{\text {collapse }}$.

The simulation of the particle loss in strongly attractive rotating and exploding BECs, with a single axial vortex of small angular momentum per particle, may stimulate further theoretical and experimental studies. We have considered small vortex states as they can be well described by the mean-field GP equation. This will provide a test for the usefulness of this equation in handling particle loss. Otherwise, a similar study with a large Bose-Einstein condensed vortex lattice $[5,6]$ is more challenging from both experimental and theoretical points.

\section{ACKNOWLEDGMENTS}

This work was supported in part by the CNPq and FAPESP of Brazil.
[1] M. H. Anderson, J. R. Ensher, Jr., M. R. Matthews, C. E. Wieman, and E. A. Cornell, Science 269, 198 (1995); J. R. Ensher, D. S. Jin, M. R. Matthews, C. E. Wieman, and E. A. Cornell, Phys. Rev. Lett. 77, 4984 (1996); K. B. Davis, M. O. Mewes, M. R. Andrews, N. J. van Druten, D. S. Durfee, D. M. Kurn, and W. Ketterle, ibid. 75, 3969 (1995); D. G. Fried, T. C. Killian, L. Willmann, D. Landhuis, S. C. Moss, D. Kleppner, and T. J. Greytak, ibid. 81, 3811 (1998).

[2] J. M. Gerton, D. Strekalov, I. Prodan, and R. G. Hulet, Nature (London) 408, 692 (2001); C. C. Bradley, C. A. Sackett, J. J. Tollett, and R. G. Hulet, Phys. Rev. Lett. 75, 1687 (1995); C. C. Bradley, C. A. Sackett, and R. G. Hulet, ibid. 78, 985 (1997)

[3] F. Dalfovo, S. Giorgini, L. P. Pitaevskii, and S. Stringari, Rev. Mod. Phys. 71, 463 (1999).

[4] K. W. Madison, F. Chevy, W. Wohlleben, and J. Dalibard, Phys. Rev. Lett. 84, 806 (2000); M. R. Matthews, B. P. Anderson, P. C. Haljan, D. S. Hall, C. E. Wieman, and E. A. Cornell, ibid. 83, 2498 (1999).

[5] K. W. Madison, F. Chevy, V. Bretin, and J. Dalibard, Phys. Rev. Lett. 86, 4443 (2001).

[6] C. Raman, J. R. Abo-Shaeer, J. M. Vogels, K. Xu, and W. Ketterle, Phys. Rev. Lett. 87, 210402 (2001); J. R. Abo-Shaeer, C. Raman, J. M. Vogels, and W. Ketterle, Science 292, 476 (2001); J. R. Abo-Shaeer, C. Raman, and W. Ketterle, Phys. Rev. Lett. 88, 070409 (2002).
[7] S. Inouye, M. R. Andrews, J. Stenger, H. J. Miesner, D. M. Stamper-Kurn, and W. Ketterle, Nature (London) 392, 151 (1998); J. Stenger, S. Inouye, M. R. Andrews, H. J. Miesner, D. M. Stamper-Kurn, and W. Ketterle, Phys. Rev. Lett. 82, 2422 (1999).

[8] Ph. Courteille, R. S. Freeland, D. J. Heinzen, F. A. van Abeelen, and B. J. Verhaar, Phys. Rev. Lett. 81, 69 (1998); S. L. Cornish, N. R. Claussen, J. L. Roberts, E. A. Cornell, and C. E. Wieman, ibid. 85, 1795 (2000).

[9] V. Vuletić, A. J. Kerman, C. Chin, and S. Chu, Phys. Rev. Lett. 82, 1406 (1999).

[10] E. Timmermans, P. Tommasini, M. Hussein, and A. Kerman, Phys. Rep. 315, 199 (1999).

[11] F. Dalfovo and S. Stringari, Phys. Rev. A 53, 2477 (1996); S. K. Adhikari, ibid. 65, 033616 (2002); E. Lundh, C. J. Pethick, and H. Smith, ibid. 58, 4816 (1998); G. M. Kavoulakis, B. Mottelson, and C. J. Pethick, ibid. 62, 063605 (2000); J. J. García-Ripoll and V. M. Pérez-García, Phys. Rev. Lett. 84, 4264 (2000).

[12] B. Jackson, J. F. McCann, and C. S. Adams, Phys. Rev. A 61, 013604 (1999).

[13] D. L. Feder, C. W. Clark, and B. I. Schneider, Phys. Rev. Lett. 82, 4956 (1999).

[14] R. J. Marshall, G. H. C. New, K. Burnett, and S. Choi, Phys. Rev. A 59, 2085 (1999). 
[15] R. Dum, J. I. Cirac, M. Lewenstein, and P. Zoller, Phys. Rev. Lett. 80, 2972 (1998).

[16] A. A. Svidzinsky and A. L. Fetter, Phys. Rev. A 62, 063617 (2000)

[17] D. L. Feder and C. W. Clark, Phys. Rev. Lett. 87, 190401 (2001); A. Aftalion and Q. Du, Phys. Rev. A 64, 063603 (2001); M. Tsubota, K. Kasamatsu, and M. Ueda, ibid. 65, 023603 (2002); Y. Castin and R. Dum, Eur. Phys. J. D 7, 399 (1999).

[18] E. P. Gross, Nuovo Cimento 20, 454 (1961); L. P. Pitaevskii, Zh. Éksp. Teor. Fiz. 40, 646 (1961) [Sov. Phys. JETP 13, 451 (1961)]; A. J. Leggett, Rev. Mod. Phys. 73, 307 (2001).

[19] R. J. Dodd, M. Edwards, C. J. Williams, C. W. Clark, M. J. Holland, P. A. Ruprecht, and K. Burnett, Phys. Rev. A 54, 661 (1996); M. Houbiers and H. T. C. Stoof, ibid. 54, 5055 (1996).

[20] J. L. Roberts, N. R. Claussen, S. L. Cornish, E. A. Donley, E. A. Cornell, and C. E. Wieman, Phys. Rev. Lett. 86, 4211 (2001).

[21] S. K. Adhikari, Phys. Rev. E 65, 016703 (2002); S. K. Adhikari and P. Murganandam, J. Phys. B 35, 2831 (2002).

[22] E. A. Donley, N. R. Claussen, S. L. Cornish, J. L. Roberts, E. A. Cornell, and C. E. Wieman, Nature (London) 412, 295 (2001).

[23] H. Saito and M. Ueda, Phys. Rev. A 63, 043601 (2001); Phys. Rev. Lett. 86, 1406 (2001); A. Eleftheriou and K. Huang, Phys. Rev. A 61, 043601 (2000).
[24] R. A. Duine and H. T. C. Stoof, Phys. Rev. Lett. 86, 2204 (2001).

[25] Y. Kagan, A. E. Muryshev, and G. V. Shlyapnikov, Phys. Rev. Lett. 81, 933 (1998); M. Ueda and K. Huang, Phys. Rev. A 60, 3317 (1999); L. Santos and G. V. Shlyapnikov, ibid. 66, 011602 (2002)

[26] H. Saito and M. Ueda, Phys. Rev. A 65, 033624 (2002).

[27] S. K. Adhikari, Phys. Rev. A 66, 013611 (2002).

[28] S. K. Adhikari, Phys. Lett. A 296, 145 (2002).

[29] J. L. Roberts, N. R. Claussen, S. L. Cornish, and C. E. Wieman, Phys. Rev. Lett. 85, 728 (2000).

[30] B. D. Esry, C. H. Greene, and J. P. Burke, Jr., Phys. Rev. Lett. 83, 1751 (1999).

[31] H. Saito and M. Ueda, e-print cond-mat/0204363.

[32] G. P. Berman, A. Smerzi, and A. R. Bishop, Phys. Rev. Lett. 88, 120402 (2002); D. S. Rokhsar, e-print cond-mat/9709212.

[33] A. J. Moerdijk, H. M. J. M. Boesten, and B. J. Verhaar, Phys. Rev. A 53, 916 (1996).

[34] S. K. Adhikari, Phys. Rev. E 62, 2937 (2000); 63, 056704 (2001); Phys. Rev. A 63, 043611 (2001).

[35] M. Holland and J. Cooper, Phys. Rev. A 53, R1954 (1996); F. Dalfovo and M. Modugno, ibid. 61, 023605 (2000); P. A. Ruprecht, M. J. Holland, K. Burnett, and M. Edwards, ibid. 51, 4704 (1995).

[36] S. E. Koonin and D. C. Meredith, Computational Physics Fortran Version (Addison-Wesley, Reading, MA, 1990), pp. 169180.

[37] E. A. Donley (private communication). 